\title{
Tree-Structured Random Vector Quantization for Beamforming in a Multiantenna Channel
}

\author{
Wiroonsak Santipach \\ Department of Electrical Engineering \\ Faculty of Engineering, Kasetsart University \\ Bangkok, Thailand 10900 \\ Email: wiroonsak.s@ku.ac.th
}

\begin{abstract}
A point-to-point multiantenna wireless channel is considered. Based on channel information, a receiver selects a transmit beamforming vector, which contains transmit antenna gains, from a vector set or codebook. The codebook index for the selected beamforming vector that maximizes channel capacity is relayed to the transmitter via a rate-limited feedback channel. Previously, we have proposed a Random Vector Quantization (RVQ) codebook, which consists of independent isotropically distributed vectors and showed that it performs close to the optimal codebook. However, RVQ requires exhaustive search to locate the desired beamformer. To lessen the search complexity, we propose a tree-structured (TS) RVQ. Numerical results show that number of computations required for TS-RVQ search can be orders of magnitude fewer than that required for RVQ search for given performance.
\end{abstract}

\section{INTRODUCTION}

Optimizing transmitter and receiver in a multiantenna system increases a channel capacity substantially [1], [2]. However, the optimization requires channel information at both the transmitter and receiver. In practice, channel estimation can be performed at the receiver with training sequence, but may not be possible at the transmitter for some system, e.g., frequency division duplex. Alternatively, the receiver, which has channel knowledge, can send channel information back to the transmitter via a feedback channel. Since the feedback channel usually supports a low data rate, channel information need to be quantized. The system performance depends on available feedback rate and design of quantization codebook, which we intend to investigate in this paper.

We are interested in quantizing a transmit beamforming vector, which contains gains for transmit antennas. References [2]-[8] have proposed different quantization schemes and codebook designs. In most feedback schemes mentioned, the receiver selects the optimal beamforming vector (e.g., maximizing capacity) from a quantization codebook, which is known a priori at both the transmitter and receiver. The codebook index for the selected vector is instantaneously relayed to the transmitter via an error-free feedback channel. This is essentially a vector quantization problem whose objective function is channel capacity. To construct the codebook, [3], [6] apply the well-known Lloyd algorithm. In [4], [5], [8], the

This work was supported by Thailand Commission on Higher Education and the Thailand Research Fund under grant MRG5080385. codebook is optimized by maximizing the minimum distance between any two vectors in Grassmannian space.

In [2], [9] , we have proposed a Random Vector Quantization (RVQ) codebook, which consists of independent isotropically distributed unit-norm vectors and showed that RVQ is optimal over all codebooks in a large system limit in which system parameters (e.g., number of antennas and feedback bits) tend to infinity with fixed ratios. For a finite-size system, RVQ is suboptimal, but performs close to the optimal codebook [2], [10]. RVQ codebook is simple to construct. However, it (including all codebook designs previously mentioned) requires an exhaustive search to locate the optimal beamforming vector. Thus, search complexity increases exponentially with number of feedback bits and becomes an issue for large number of feedback bits.

To reduce the search complexity, here we propose to modify RVQ codebook with binary tree structure. The search complexity of a tree-structured codebook increases linearly with feedback bits. In building the tree, the generalized Lloyd algorithm (GLA) is used to cluster the codebook entries. We note that the selected vector from this tree-structured (TS)RVQ may not be the vector that maximizes the capacity. Thus, the performance of TS-RVQ will be worse than that of the original RVQ. However, numerical examples show that the performance gap between TS-RVQ and RVQ is small. Furthermore, number of computations needed for TS-RVQ search can be orders of magnitude fewer than that for RVQ search for given capacity. There are other work [7], [11], which consider simpler search or encoding schemes. In [11], tree structure was considered. However, codebook entries are obtained from iterative Lloyd algorithm, which incurs additional complexity. The search algorithm for a QAM-based codebook was proposed by [7] and is based on a noncoherent sequence detection algorithm. The search complexity of this scheme also grows linearly with feedback bit.

\section{SYSTEM MODEL}

Here we consider a point-to-point Rayleigh fading channel with $N_{t}$ transmit antennas and $N_{r}$ receive antennas. For a discrete-time model, the received $N_{r} \times 1$ vector is given by

$$
\boldsymbol{r}=\boldsymbol{H} \boldsymbol{v} x+\boldsymbol{n}
$$


where $\boldsymbol{H}=\left[h_{i j}\right]$ is an $N_{r} \times N_{t}$ channel matrix whose element $h_{i j}$ is the channel coefficient between the $j$ th transmit and the $i$ th receive antennas, $\boldsymbol{v}$ is an $N_{t} \times 1$ beamforming vector containing transmit antenna gains, $x$ is a transmitted symbol with zero mean and unit variance, and $\boldsymbol{n}$ is an $N_{r} \times 1$ additive white Gaussian noise vector with zero mean and covariance $\sigma_{n}^{2} \boldsymbol{I}$ and $\boldsymbol{I}$ is an identity matrix. Assuming ideal scattering and Rayleigh fading, $h_{i j}$ is independent and complex Gaussian distributed with zero mean and unit variance. In [2], $v$ is generalized to a precoding matrix, which multiplexes different data streams onto transmit antennas. Here we consider a precoding or beamforming vector with one data stream. The channel capacity is given by

$$
C=E_{\boldsymbol{H}}\left[\log \left(1+\rho \boldsymbol{v}^{\dagger} \boldsymbol{H}^{\dagger} \boldsymbol{H} \boldsymbol{v}\right)\right]
$$

where the expectation is over distribution of $\boldsymbol{H}$, and $\rho=1 / \sigma_{n}^{2}$ is the background signal-to-noise ratio (SNR).

The receiver, which is assumed to know $\boldsymbol{H}$, can optimize the capacity over the transmit beamforming vector $\boldsymbol{v}$. The optimal $\boldsymbol{v}$ is the eigenvector of $\boldsymbol{H}^{\dagger} \boldsymbol{H}$, corresponding to the maximum eigenvalue. Ideally, the receiver relays the optimal beamforming vector to the transmitter without quantization. In practical system, the feedback channel between the receiver and the transmitter is rate-limited. Thus, the receiver needs to quantize the beamforming vector and sends back the quantized vector.

Since eigenvector of $\boldsymbol{H}^{\dagger} \boldsymbol{H}$ is isotropically distributed, this motivated us to propose an RVQ codebook, which consists of independent isotropically distributed vectors [2], [9]. With $B$ bits, the RVQ codebook $\mathcal{V}=\left\{\boldsymbol{v}_{1}, \ldots, \boldsymbol{v}_{2^{B}}\right\}$. The receiver selects

$$
\begin{aligned}
\hat{\boldsymbol{v}} & =\arg \max _{1 \leq i \leq 2^{B}} \log \left(1+\rho \boldsymbol{v}_{i}^{\dagger} \boldsymbol{H}^{\dagger} \boldsymbol{H} \boldsymbol{v}_{i}\right) \\
& =\arg \max _{1 \leq i \leq 2^{B}} \boldsymbol{v}_{i}^{\dagger} \boldsymbol{H}^{\dagger} \boldsymbol{H} \boldsymbol{v}_{i}
\end{aligned}
$$

and the associated capacity

$$
C_{\mathrm{rvq}}=E_{\boldsymbol{H}, \mathcal{V}}\left[\log \left(1+\rho \hat{\boldsymbol{v}}^{\dagger} \boldsymbol{H}^{\dagger} \boldsymbol{H} \hat{\boldsymbol{v}}\right)\right]
$$

where the expectation is over both distributions of $\boldsymbol{H}$ and $\mathcal{V}$. It was shown in [2], [12] that RVQ is optimal in a large system limit in which $N_{t}, N_{r}, B$ tend to infinity with fixed normalized number of receive antennas $N_{r} / N_{t}$ and normalized feedback $B / N_{t}$. The closed-form expression for (5) in a large system limit is obtained in [2]. For a finite-size system, RVQ was shown in [2], [10] to perform close to the optimized codebook proposed in [5].

Although generating RVQ codebook is relatively straightforward, locating the optimal entry in the codebook requires exhaustive search. Since number of codebook entries increases exponentially with $B$, so does the search complexity.

\section{TREE-STRUCTURED RVQ}

To motivate the proposed codebook design, we first consider the inner product $\hat{\boldsymbol{v}}^{\dagger} \boldsymbol{H}^{\dagger} \boldsymbol{H} \hat{\boldsymbol{v}}$, which can be bounded as follows
[7]

$$
\lambda_{1}\left|\boldsymbol{s}_{1}^{\dagger} \hat{\boldsymbol{v}}\right|^{2} \leq \hat{\boldsymbol{v}}^{\dagger} \boldsymbol{H}^{\dagger} \boldsymbol{H} \hat{\boldsymbol{v}} \leq \lambda_{1}\left|\boldsymbol{s}_{1}^{\dagger} \hat{\boldsymbol{v}}\right|^{2}+\lambda_{2} \sum_{i=2}^{N_{t}}\left|\boldsymbol{s}_{i}^{\dagger} \hat{\boldsymbol{v}}\right|^{2}
$$

where $\lambda_{1} \geq \lambda_{2} \geq \ldots \geq \lambda_{N_{t}}$ are ordered eigenvalues of $\boldsymbol{H}^{\dagger} \boldsymbol{H}$ and $s_{i}$ 's are eigenvectors associating with eigenvalues $\lambda_{i}$ 's. As $B \rightarrow \infty$, both bounds converge to the maximum eigenvalue $\lambda_{1}$ and the optimal beamforming $\hat{\boldsymbol{v}} \rightarrow \boldsymbol{s}_{1}\left(\left\|\hat{\boldsymbol{v}}-\boldsymbol{s}_{1}\right\| \rightarrow 0\right)$. For finite $B$, the beamforming vector $\boldsymbol{v}$ that is closest in Euclidean distance to $s_{1}$ is not optimum since it may not maximize the capacity or, equivalently $\boldsymbol{v}^{\dagger} \boldsymbol{H}^{\dagger} \boldsymbol{H} \boldsymbol{v}$. However, [7] showed that selecting the beamformer that is closest to $s_{1}$ performs close to selecting with (4). This motivates us to use Euclidean distance in constructing the codebook tree.

We start with RVQ codebook $\mathcal{V}$ with $2^{B}$ independent isotropically distributed vectors. In $N_{t}$-dimensional space, these vectors (points) are uniformly distributed on a surface of the unit ball. To generate each vector entry, we generate vector whose elements are independent identically distributed (i.i.d.) and normalize it. For a binary tree, we iteratively find two centroids $c_{1}$ and $c_{2}$ that minimize sum of Euclidean distances between vectors to the centroids,

$$
d=\sum_{v_{j} \in \mathcal{V}_{1}}\left\|\boldsymbol{c}_{1}-\boldsymbol{v}_{j}\right\|+\sum_{v_{k} \in \mathcal{V}_{2}}\left\|\boldsymbol{c}_{1}-\boldsymbol{v}_{k}\right\|
$$

where the two quantization regions or clusters are given by

$$
\mathcal{V}_{1}=\left\{\boldsymbol{v}_{j} \in \mathcal{V}:\left\|c_{1}-\boldsymbol{v}_{j}\right\| \leq\left\|\boldsymbol{c}_{2}-\boldsymbol{v}_{j}\right\|\right\}
$$

and

$$
\mathcal{V}_{2}=\mathcal{V} \backslash \mathcal{V}_{1}
$$

We can iterate between computing the sum distance $d$ and finding the clusters until $d$ converges. This is the generalized Lloyd algorithm (GLA) [13], which is more precisely stated as follows.

\section{Generalized Lloyd Algorithm}

1: Start with initial centroids $\boldsymbol{c}_{1}^{(0)}, \boldsymbol{c}_{2}^{(0)}$, RVQ codebook $\mathcal{V}=$ $\left\{\boldsymbol{v}_{1}, \ldots, \boldsymbol{v}_{2^{B}}\right\}$, and the distance metric $d^{(0)}=0$. Set $k=$ 0 .

\section{2: repeat}

3: $\quad$ Find

$$
\begin{aligned}
& \mathcal{V}_{1}^{(k)}=\left\{\boldsymbol{v}_{i} \in \mathcal{V} \mid\left\|\boldsymbol{c}_{1}^{(k)}-\boldsymbol{v}_{i}\right\| \leq\left\|\boldsymbol{c}_{2}^{(k)}-\boldsymbol{v}_{i}\right\|\right\} \\
& \mathcal{V}_{2}^{(k)}=\mathcal{V} \backslash \mathcal{V}_{1}^{(k)}
\end{aligned}
$$

4: Compute

$$
d^{(k)}=\sum_{v_{j} \in \mathcal{V}_{1}^{(k)}}\left\|\boldsymbol{c}_{1}^{(k)}-\boldsymbol{v}_{j}\right\|+\sum_{v_{k} \in \mathcal{V}_{2}^{(k)}}\left\|\boldsymbol{c}_{2}^{(k)}-\boldsymbol{v}_{k}\right\| .
$$

5: Compute new centroids

$$
\begin{aligned}
& \boldsymbol{c}_{1}^{(k+1)}=\sum_{\boldsymbol{v}_{i} \in \mathcal{V}_{1}^{(k)}} \boldsymbol{v}_{i} /\left|\mathcal{V}_{1}^{(k)}\right| \\
& \boldsymbol{c}_{2}^{(k+1)}=\sum_{\boldsymbol{v}_{j} \in \mathcal{V}_{2}^{(k)}} \boldsymbol{v}_{j} /\left|\mathcal{V}_{2}^{(k)}\right|
\end{aligned}
$$


where $\left|\mathcal{V}_{i}^{(k)}\right|$ denotes number of vectors in the set.

6: $\quad k=k+1$

7: until $\frac{d^{(k)}-d^{(k-1)}}{d^{(k)}}<\epsilon$

Then, we divide $\mathcal{V}_{1}$ and $\mathcal{V}_{2}$ each into two clusters and find two pairs of centroids. We repeat this process until we obtain a tree whose leaf node contains only one vector entry from the RVQ codebook. The steps are summarized as follows.

Tree Building Algorithm

1: Generate RVQ codebook with $2^{B}$ entries.

2: Store the entire codebook at the root of a binary tree.

3: repeat

4: Use the GLA to obtain the two centroids $\boldsymbol{c}_{1}$ and $\boldsymbol{c}_{2}$ and the corresponding two clusters.

5: Create two child nodes and store each subset in each child node.

6: until Each leaf node contains only one vector.

We note that with this algorithm, the tree produced may not be balanced. As $B$ increases, complexity of building the tree increases significantly. But, it should not incur any additional delay since the tree can be obtained offline.

To find the optimal vector, we start at the root node and transverse the tree by comparing the inner products $\boldsymbol{c}_{1}^{\dagger} \boldsymbol{H}^{\dagger} \boldsymbol{H} \boldsymbol{c}_{1}$ and $\boldsymbol{c}_{2}^{\dagger} \boldsymbol{H}^{\dagger} \boldsymbol{H} \boldsymbol{c}_{2}$ where $\boldsymbol{c}_{1}$ and $\boldsymbol{c}_{2}$ are centroids of the vector sets for the left and right child nodes, respectively. The steps are stated as follows:

Encoding Algorithm

1: Start at the root node with input channel matrix $\boldsymbol{H}$.

2: while The vector set for the node has more than one vector. do

3: $\quad$ if $\boldsymbol{c}_{1}^{\dagger} \boldsymbol{H}^{\dagger} \boldsymbol{H} \boldsymbol{c}_{1} \geq \boldsymbol{c}_{2}^{\dagger} \boldsymbol{H}^{\dagger} \boldsymbol{H} \boldsymbol{c}_{2}$ then

4: $\quad$ Move to the left child node.

5: $\quad$ else

6: $\quad$ Move to the right child node.

7: $\quad$ end if

8: end while

9: return The vector in the set.

For multi-input single-output (MISO) channel, $\boldsymbol{H}$ is a $1 \times N_{t}$ vector. The objective is simplified to finding the vector with closest Euclidean distance and the problem becomes a classical vector quantization, which is well investigated (see [14] and references therein).

\section{NumericAl Results}

To implement the GLA, we use function kmeans in Matlab. kmeans uses two-phase iterative algorithm. The first phase is batch update, which is the GLA stated in Section III. The second phase is online update. In this phase, each vector is reassigned to the other cluster if doing so will result in lower sum distance.

Fig. 1 shows channel capacity for MISO channel versus number of feedback bits per transmit antenna with $N_{t}=2$ and 3. We compare the performance of TS-RVQ with the original RVQ and show that TS-RVQ performs a bit worse than RVQ as expected. The maximum performance gap is less than $5 \%$ for both channels. We note that with 4 bits per complex coefficients, both RVQ schemes perform close to the unlimited feedback $(B \rightarrow \infty)$ capacity for $N_{t}=2$. Since number of entries in RVQ codebook increases exponentially with $B$, we only have results for moderate $B$ for $N_{t}=3$.

In Fig. 2, we show capacity of MISO channels with number of inner product computations needed for each scheme. For RVQ, the quantizer needs to compute inner product $\boldsymbol{v}_{i}^{\dagger} \boldsymbol{H}^{\dagger} \boldsymbol{H} \boldsymbol{v}_{i}$ $2^{B}$ times. For TS-RVQ, two inner product computations are required for each node transversed. The total number of computations for TS-RVQ depends on the tree's depth and increases linearly with $B$. For a capacity close to the unlimited feedback capacity, number of computations needed for TSRVQ can be substantially fewer than that for RVQ. For $N_{t}=3$ and $C=5.25$ bits per channel use, TS-RVQ needs 30 computations while RVQ needs more than 1000 computations. We note that the gap widens as number of feedback bits increases.

Figs. 3 and 4 show performance of RVQ and TS-RVQ for multi-input multi-output (MIMO) channels. Similar to Fig. 1, capacity in Fig. 3 increases with number of available feedback bits. Given $B / N_{t}$, the capacity of MIMO channel is greater than that of MISO channel due to spatial diversity at the receiver. Similar to the MISO channel, a capacity gap between RVQ and TS-RVQ is small for given feedback. However, a difference between search computations can be large. For the $4 \times 3$ system and $C=7.25$ bits per channel use, TS-RVQ requires roughly 30 computations while RVQ requires about 400 computations.

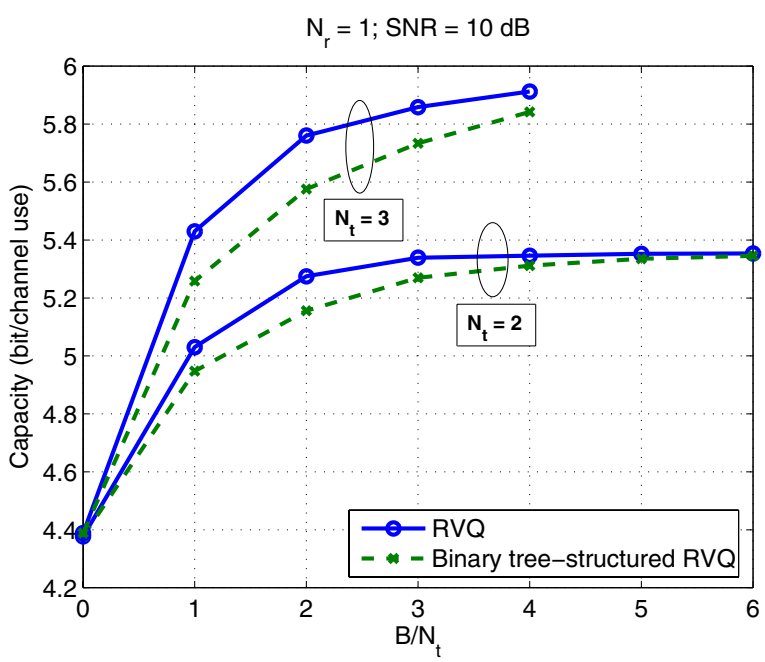

Fig. 1. Shown is capacity for MISO channels with quantized beamforming versus feedback bit $B$. Performance of RVQ and TS-RVQ are compared with $N_{t}=2$ and 3 , and $\mathrm{SNR}=10 \mathrm{~dB}$.

\section{Conclusions}

To quantize a transmit beamforming vector, we have proposed tree-structured RVQ. Compared with the original RVQ, TS-RVQ gives lower channel capacity for given feedback bits. However, the performance difference is minimal. For search complexity, we compared number of inner product 


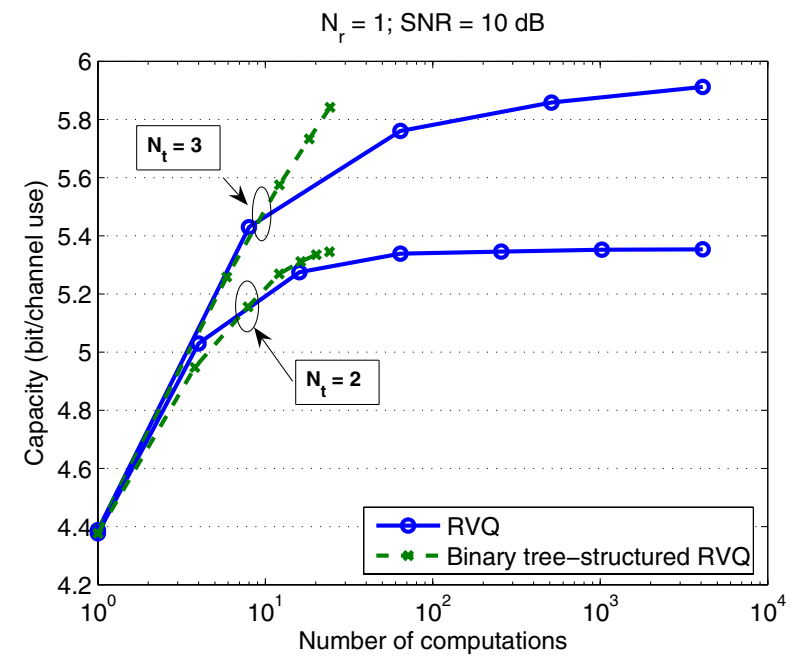

Fig. 2. Capacity for MISO channels with quantized beamforming is shown with number of inner product computations performed during quantization encoding. Performance of RVQ and TS-RVQ are compared for SNR $=10$ $\mathrm{dB}$.

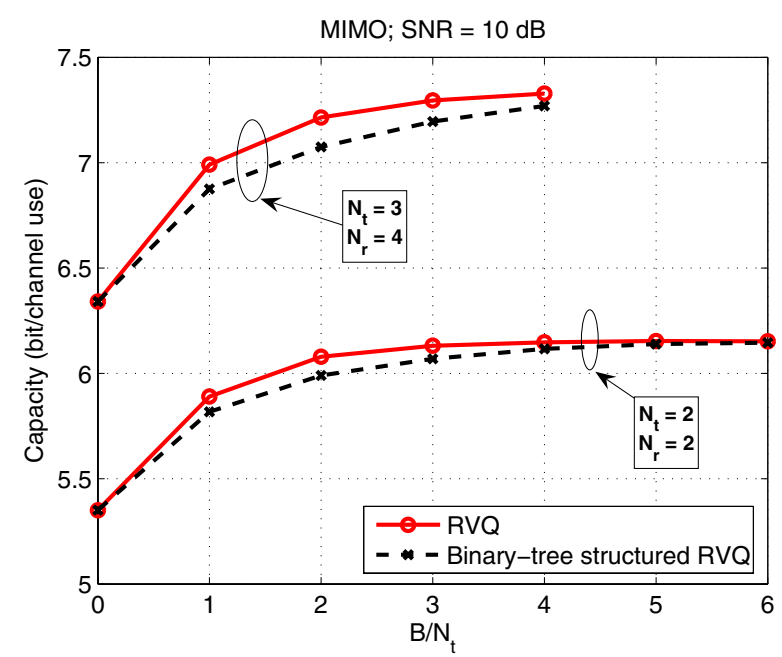

Fig. 3. Shown is capacity of beamforming for MIMO channels with feedback bit $B$. Performance of RVQ and TS-RVQ for $2 \times 2$ and $3 \times 4$ channels are compared.

computations and showed that number of computations needed for TS-RVQ can be orders of magnitude lower than that for RVQ. As number of feedback bits increases, the difference in search complexity increases significantly. Although search computation for TS-RVQ is less complex, building a codebook tree for large $B$ can be computationally intense. Fortunately, tree construction can be performed offline. Here we only consider beamforming and point-to-point link. Future research problems include extending algorithms to transmit precoding matrix, and multi-user channel. Also we only showed numerical examples and thus, some performance analysis for TSRVQ is of interest.

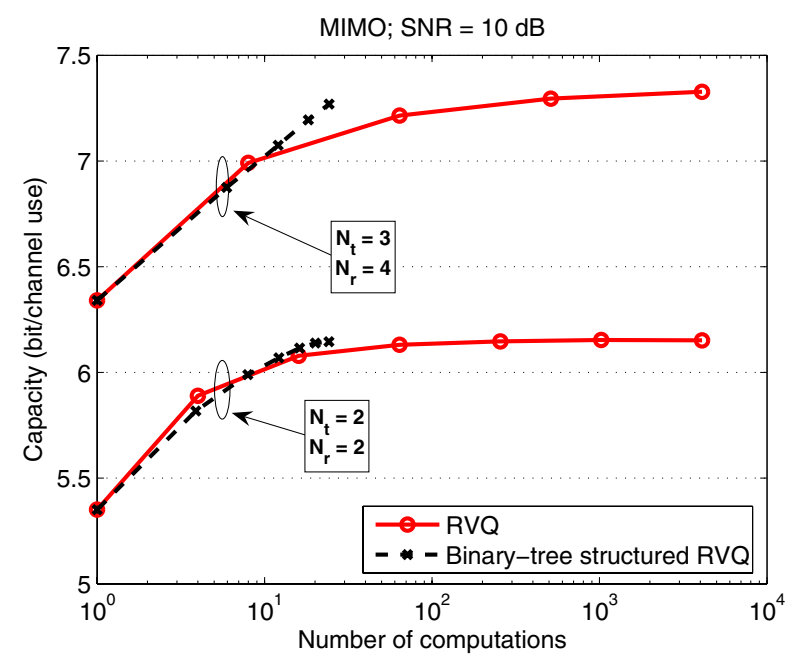

Fig. 4. Capacity of beamforming for MIMO channels is shown with number of inner product computations performed during quantization encoding.

\section{REFERENCES}

[1] I. E. Telatar, "Capacity of multi-antenna Gaussian channels," European Trans. on Telecommun., vol. 10, pp. 585-595, Nov. 1999.

[2] W. Santipach and M. L. Honig, "Capacity of a multi-antenna fading channel with a quantized precoding matrix," IEEE Trans. Inf. Theory, Dec. 2006, submitted for publication.

[3] A. Narula, M. J. Lopez, M. D. Trott, and G. W. Wornell, "Efficient use of side information in multiple antenna data transmission over fading channels," IEEE J. Sel. Areas Commun., vol. 16, no. 8, pp. 1423-1436, Oct. 1998.

[4] K. K. Mukkavilli, A. Sabharwal, E. Erkip, and B. Aazhang, "On beamforming with finite rate feedback in multiple antenna systems," IEEE Trans. Inf. Theory, vol. 49, no. 10, pp. 2562-2579, Oct. 2003.

[5] D. J. Love and R. W. Heath, Jr., "Grassmannian beamforming for multiple-input multiple-output wireless systems," IEEE Trans. Inf. Theory, vol. 49, no. 10, pp. 2735-2745, Oct. 2003.

[6] V. K. N. Lau, Y. Liu, and T.-A. Chen, "On the design of MIMO blockfading channels with feedback-link capacity constraint," IEEE Trans. Commun., vol. 52, no. 1, pp. 62-70, Jan. 2004.

[7] D. J. Ryan, I. V. L. Clarkson, I. B. Collings, D. Guo, and M. L. Honig, "QAM and PSK codebooks for limited feedback MIMO beamforming," in Proc. IEEE Int. Conf. on Commun. (ICC), Glasgow, Scotland, Jun. 2007.

[8] J. C. Roh and B. D. Rao, "Transmit beamforming in multiple-antenna systems with finite rate feedback: A VQ-based approach," IEEE Trans. Inf. Theory, vol. 52, no. 3, pp. 1101-1112, Mar. 2006.

[9] W. Santipach and M. L. Honig, "Signature optimization for DS-CDMA with limited feedback," in Proc. IEEE Int. Symp. on Spread-Spectrum Tech. and Appl. (ISSSTA), Prague, Czech Republic, Sep. 2002, pp. 180 184.

[10] D. J. Love, R. W. Heath, Jr., W. Santipach, and M. L. Honig, "What is the value of limited feedback for MIMO channels?" IEEE Commun Mag., vol. 42, no. 10, pp. 54-59, Oct. 2004.

[11] J. C. Roh and B. D. Rao, "Vector quantization techinques for multipleantenna channel information feedback," in IEEE Intl. Conf. on Signal Processing \& Commun. (SPCOM), Bangalore, India, Dec. 2004, pp. 402-406.

[12] W. Santipach and M. L. Honig, "Asymptotic capacity of beamforming with limited feedback," in Proc. IEEE Int. Symp. on Info. Theory (ISIT), Chicago, IL, Jun. 2004, p. 290.

[13] A. Gersho and R. M. Gray, Vector Quantization and Signal Compression. Boston: Kluwer, 1992.

[14] I. Katsavounidis, C.-C. J. Kuo, and Z. Zhang, "Fast tree-structured nearest neighbor encoding for vector quantization," IEEE Trans. Image Process., vol. 5, no. 2, pp. 398-404, Feb. 1996. 